In $100 \mathrm{~g}$

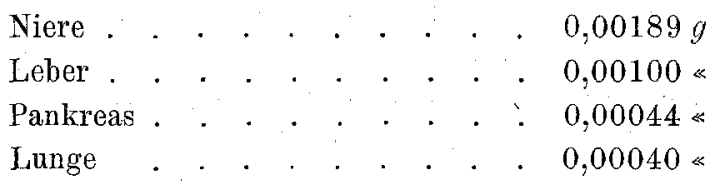

\title{
Benzol als Indikator für die Jodometrie.
}

\section{B. Schwezow.}

Das Blauwerden der Stärke unter der Einwirkung von Jod ist eine so empfindliche Reaktion, dass es ganz überflüssig scheint, neue Indikatoren zu suchen. Es gibt aber Fälle, wo die Empfindlichkeit der oben genannten Reaktion nicht genügt. Einen solchen Fall hatte ich vorigen Sommer, während meiner Arbeit im Leipziger physikalischchemischen Institut aufzuweisen, als ich eine ganze Reihe von Chlorbestimmungen in Benzollösungen auf jodometrischem Wege zu machen hatte. Da die ausgeschiedene Jodmenge sehr gering und das Jod zwischen zwei Lösungsmitteln verteilt war, so wurde das. Titrieren in Fläschchen mit eingeschliffenen Glasstöpseln unter beständigem Schütteln ausgeführt und die gebrauchten Titrierflüssigkeiten sehr verdünnt (etwa $1 / 150$-normal). Das erste Erscheinen der Färbung ist viel leichter zu bemerken als ihr Verschwinden, und deshalb wandte ich die Methode des Zurücktitrierens an. Unter diesen Bedingungen erwies sich die Jodstärkereaktion als wenig empfindlich; die Färbung erschien äusserst undeutlich und war rötlich-violett; sie ging nur bei grossem Zusatz von Jod in blau über. Die Beendigung der Reaktion genau zu bestimmen, war sehr schwer. Da fiel es mir ein, unter diesen Bedingungen das Benzol selbst als Indikator anzuwenden, da das Jod sich in demselben mit intensiv roter Färbung auflöst.

Versuche in dieser Richtung wurden im chemischen Laboratorium der Moskauer Gewerbe-Schule angestellt, wobei ich beschloss, ausser Benzol auch andere, gewöhnlich bei Entdeckungen geringer Jodmengen gebrauchte Lösungsmittel (Schwefelkohlenstoff, Chloroform etc.) anzuwenden.

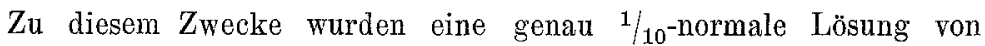

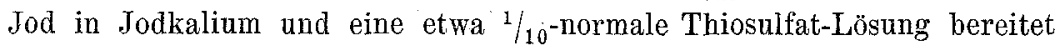


und ihr Verhältnis zu einander bestimmt, wobei als Indikator bald Stärkekleister, bald Benzol angewandt wurde. Die Resultate sind in der folgenden Tabelle I verzeichnet, wobei die Ziffern die Zahl der Kubikzentimeter einer ${ }^{n} / 10$-Jodlösung bedeuten, welche zum Titrieren von $10 \mathrm{cc}$ der Thiosulfat-Lôsung verbraucht wurden.

Tabelle I.

\begin{tabular}{c|c|c}
\hline & \multicolumn{2}{|c}{ Indikator: } \\
\hline No. & $\begin{array}{c}\text { Stärke } \\
\text { (Lösliche Stärke }\end{array}$ & Benzol \\
& von Schering) & $c c$ \\
\hline 1 & $c c$ & $c$ \\
\hline 2 & 10,49 & 10,49 \\
3 & 10,49 & 10.50 \\
4 & 10,48 & 10,48 \\
5 & 10,48 & 10,49 \\
Mittel & 10,49 & - \\
\hline & 10,49 & 10,49 \\
\hline
\end{tabular}

Der Augenblick der Reaktionsbeendigung ist scharf $z u$ erkennen. Der Unterschied bei Anwendung beider Indikatoren liegt in den Grenzen der Beobachtungsfehler, so dass man die Ergebnisse als übereinstimmend betrachten kann. Um diesen Unterschied hervorzuheben, wurden noch verdünntere Lösungen zubereitet (eine genau $1 / 100$-normale Jodlösung und eine etwa $1 / 100^{-n o r m a l e ~ T h i o s u l f a t-L o ̈ s u n g) ~ u n d ~ w i e d e r ~ d i e ~ A n z a h l ~ K u b i k-~}$ zentimeter Jodlösung bestimmt, welche zum Titrieren von $10 \mathrm{cc}$ ThiosulfatLösung verbraucht wurde. Die folgende Tabelle II gibt die Resultate:

Tabelle II.

\begin{tabular}{|c|c|c|}
\hline \multirow[b]{2}{*}{ No. } & \multicolumn{2}{|c|}{ Indikator: } \\
\hline & $\begin{array}{c}\text { Stärke } \\
\text { (Lösliche Stärke } \\
\text { von Schering) } \\
c c\end{array}$ & $\begin{array}{l}\text { Benzol } \\
\quad c c\end{array}$ \\
\hline 1 & 10,53 & 10,53 \\
\hline 2 & 10,54 & 10,51 \\
\hline 3 & 10,55 & 10,52 \\
\hline 4 & 10,53 & 10,53 \\
\hline Mittel & 10,54 & 10,52 \\
\hline
\end{tabular}


Das Ende der Reaktion bei Anwendung von Benzol ist deutlich ausgedrückt, bei Anwendung von Stärke hingegen undeutlich. Der Unterschied beim Gebrauch dieser Indikatoren kann hier nicht durch Beobachtungsfehler erklärt werden und beweist die grössere Empfindlichkeit des Benzols im Vergleiche zur Stärke.

Um die Frage von der Empfindlichkeit der verschiedenen Indikatoren endgültig festzustellen und vergleichbare Ziffern zu erhalten, wurde eine sehr verdünnte Jodlösung bereitet ( $1 / 10000$ normal), und die Empfindlichkeit der verschiedenen Indikatoren durch die Zahl der Kubikzentimeter dieser zum Hervortreten der deutlichen Färbung nötigen Lösung ausgedrückt.

Bei allen diesen Versuchen wurde dieselbe Quantität $(5 c c)$ der verschiedenen Indikatoren angewandt und $10 \mathrm{cc}$ Wasser hinzugefügt. Die Temperatur dabei betrug etwa $+15^{\circ} \mathrm{C}$. Da die Dissoziation der Jodstärkeverbindung bei gewöhnlicher Temperatur sehr gross ist, so wurden die Versuche mit Stärke ausser bei einer Temperatur von $+15^{0} \mathrm{C}$. auch noch bei $+5^{\circ} \mathrm{C}$. ausgeführt.

Die in folgender Tabelle III gesammelten Zahlen stellen die Mittelwerte von vielen Beobachtungen dar.

Tabelle IUI.

\begin{tabular}{lll|c|c}
\hline Indikator & $\begin{array}{c}\text { Kaum } \\
\text { sichtbare } \\
\text { Färbung }\end{array}$ & $\begin{array}{c}\text { Dent } \\
\text { liche } \\
\text { Färbung }\end{array}$ \\
\hline & & & & \\
\hline
\end{tabular}

Aus dieser Tabelle ergibt sich, dass Benzol der empfindlichste Indikator ist. Die Empfindlichkeit der Stärke ist also bedeutend geringer, als die des Benzols, und wird noch bei Hinzufügung von letzterem vermindert. Folgender Versuch kann als Beweis des Gesagten dienen. Es wurden $10 c c$ Wasser genommen und $5 c c$ Stärkelösung, wie auch $5 c c$ Benzol, hinzugefügt. Zum Hervortreten deutlicher hellroter Färbung des Benzols 
88. จ. Knorre: Über die Verwendung von Persulfat zu quantit. Trennungen.

waren $2 c c$ n/10000-Jodlösung nötig; die Stärke jedoch nahm erst bei Hinzufügung von 5-6cc derselben Lösung eine schwach violette Färbung an. Der Versuch wurde zweimal mit denselben Resultaten wiederholt.

Ein grosser Missstand bei der Anwendung von Benzol und der anderen oben genannten Lösungsmittel als Indikatoren liegt darin, dass man das Titrieren in gläsernen Fläschchen mit eingeschliffenen Glasstöpseln unter beständigem Schütteln vornehmen muss. Dieses Schütteln aber ist unumgänglich notwendig, wenn das in einem der oben genannten Lösungsmittel enthaltene Jod bestimmt werden soll - ein bei photochemischen Reaktionen ziemlich häufiger Fall. Unter diesen Umständen ist das Titrieren ohne Stärke durchaus vorzuziehèn, indem man sich des Lösungsmittels, als Indikator bedient.

Moskau, Gewerbe-Schule, Chemisches Laboratorium, November 1904.

Über die Verwendung von Persulfat zu quantitativen Trennungen. (Antwort an Herrn Prof. Dr. M. Dittrich.)

Von

G. v. Knorre.

In dieser Zeitschrift 43, 1 bis 14 (1904) habe ich durch eine grössere Anzahl von Versuchen den Nachweis dafür erbracht, dass sich die Fällung des Mangans durch Ammoniumpersulfat für gewichtsanalytisch'e Trennungen nicht verwenden lässt.

Ich war schon im Jahre 1901 auf Grund damals ausgeführter Versuche zu dem gleichen Schlusse gelangt ${ }^{1}$ ); da aber im Gegensatz dazu M. Dittrich und K. Hassel in mehreren 1902, und 1903 erschienenen Veröffentlichungen ${ }^{2}$ ) gerade die gewichtsanalytische Trennung des Mangans vom Kalzium, Magnesium, Zink, Kadmium, Nickel, Aluminium, Chrom und Kupfer durch Fällung mit Persulfat empfehlen, so hielt ich es für geboten, weitere diesbezügliche Versuche vorzunehmen.

Meine in dieser Zeitschrift (a. a. 0.) beschriebenen, genau nach den Angaben von Dittrich und Hassel ausgeführten Versuche zeigten, dass es vollkommen ausgeschlossen ist, insbesondere Mangan und Kupfer auch nur einigermalsen scharf

1) Zeitschrift $f$. angew. Chemie 14, 1154, 1161 (1901). s. 284,1423 .

2) Ber. d. deutsch. chem. Gesellsch. zu Berlin 1902, S. 3266, 4072; 1903, 